\title{
Adaptive Damping Control Strategy of Wind Integrated Power System
}

\author{
Jun Deng ${ }^{1, *}{ }^{\mathbb{C}}$, Jun Suo ${ }^{1}$, Jing Yang ${ }^{2}$, Shutao Peng ${ }^{1}$, Fangde Chi ${ }^{3}$ and Tong Wang ${ }^{2, *}$ \\ 1 State Grid Shaanxi Electric Power Research Institute, Xi'an 710100, China; powersuo@163.com (J.S.); \\ newpking@126.com (S.P.) \\ 2 School of Electrical \& Electronic Engineering, North China Electric Power University, Changping District, \\ Beijing 102206, China; yangjing@ncepu.edu.cn \\ 3 State Grid Shaanxi Electric Power Company, Xi'an 710048, China; chifangde@163.com \\ * Correspondence: d.jone@163.com or dengjun@dky.sn.sgcc.com.cn (J.D.); hdwangtong@ncepu.edu.cn (T.W.); \\ Tel.: +86-2989698550 (J.D.); +86-1061771407 (T.W.)
}

Received: 9 November 2018; Accepted: 10 December 2018; Published: 1 January 2019

\begin{abstract}
Random variation of grid-connected wind power can cause stochastic variation of the power system operating point. This paper proposes a new scheme to design an adaptive damping controller by tracking the variation of system operating points and updating the controller's functions to achieve a robust damping control effect. Firstly, the operating space is classified into different modes according to the classification of wind power outputs. Multiple power system stabilizers (PSSs) are then designed. Secondly, the method of optimal classification and regression decision tree (CART) is utilized for classifying subspaces of system operating point and it is proposed that the on-line measurements from wide area measurement system (WAMS) are used for tracking the dynamic behaviors of stochastic drifting point and thus guide the updating of appropriate PSSs be switched on adaptively. A 16-generator-68-bus power system integrated with wind power is presented as a test system to demonstrate that the adaptive control scheme by use of the CART can damp multi-mode oscillations effectively when the wind power output changes.
\end{abstract}

Keywords: stochastic power system operating point drift; wind integrated power system; power oscillations; adaptive damping control

\section{Introduction}

Traditional power generation is controllable and predictable. The stability and control of the power system is determined for a given set of circumstances [1]. With the rapid growth of wind power resources, the stochastic flotation of wind power has become more and more significant and cannot be neglected [2,3]. In most cases, lack of robustness of the controllers designed for the deterministic operating point when the wind power output changes a lot has become a dominant problem [4]. In a future power system, if the scale of grid-connected wind generation is comparable to the traditional power generation, system stability analysis and control has to consider the random variation features of wind power when the system operating point varies stochastically [5-7].

Therefore, as far as power system stability control is concerned, to guarantee the robust performances in the case of stochastic variation of system operating point as affected by variable integrated wind power, it is important to investigate the design of adaptive damping controller which can accommodate the stochastic variation of the system operating point. Robust control [8] is an effective way to deal with the uncertainties, while when the operating conditions change over a wide range, the robustness might not be guaranteed. Therefore, adaptive control methods such as the fuzzy control method [9], multiple-model method [10,11], Kalman filter method [12], etc., which can track 
the stochastic behavior of systems have become more emphasized. However, when the operating conditions change a lot, the nonlinearity of power systems is more prominent and the disturbance models more difficult to interpret [13]. Therefore, machine study methods using statistical analysis may be more suitable for this particular application. For example, the classification and regression tree (CART) [14], neural network method [15,16] and so on. Therefore, this paper presents an adaptive control scheme based on CART for a wind integrated power system to accommodate the stochastic variation of wind generation.

The rest part of the paper is organized as follows: firstly, the operating space is classified into different modes according to the classification of wind power outputs. On the basis of the subspaces, multiple PSSs are designed in coordination for mapping rules. Secondly, the CART is formed, trained and tested to identify the subspaces with the help of wide area measurement system (WAMS). The CART determines the selection of appropriate damping control adaptively according to the identification of the subspaces of system operating point. Finally, the results of a 16-generator 68-bus example power system are presented to verify that the proposed adaptive control scheme in the paper can effectively handle the stochastic variation of system operating point and suppress system low-frequency power oscillations when grid-connected wind power varies.

\section{The CART-Based Adaptive Damping Control Scheme}

\subsection{The Formation of Subspaces}

The subspaces are formed by dividing the wind power outputs which could cause the changing operating conditions. The lower and higher boundaries correspond to the minimum and maximum wind power outputs as shown in Figure 1a, respectively. The partition method has been used widely in power load prediction as a simple and effective approach. The stochastic wind power outputs are divided into several intervals as shown in Figure 1b, thus the subspaces are formed. All of the subspaces compose the whole operating space and they have the relation: $B_{1} \cup B_{2} \cup \ldots \cup B_{i} \cup \ldots \cup B_{n} \equiv B$. Based on the subspaces, the mapping rules are built for each subspace.

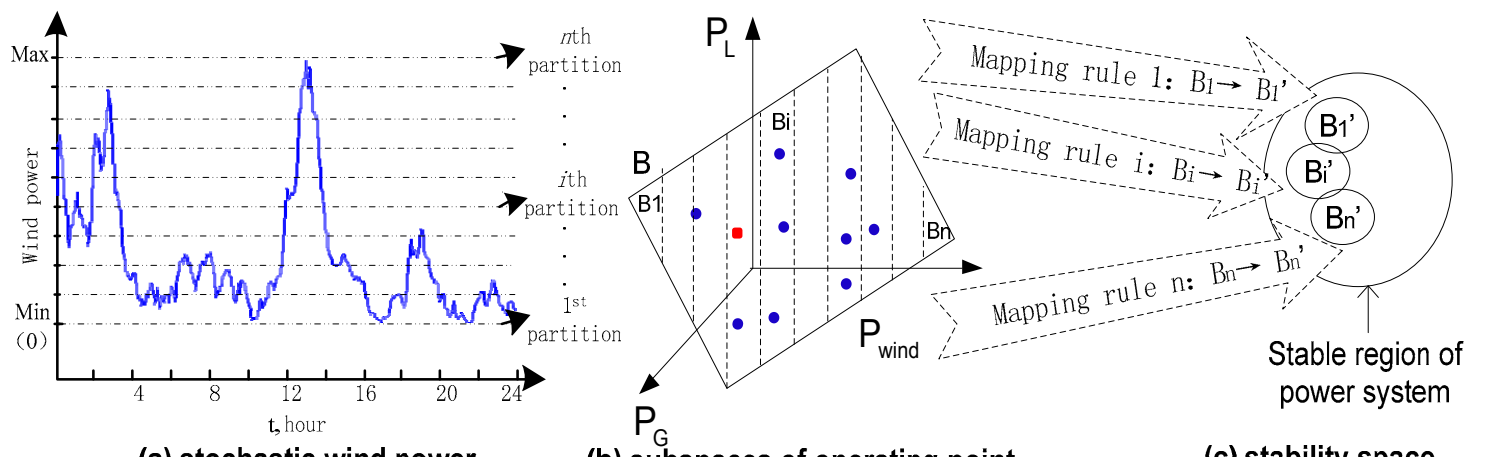

(a) stochastic wind power

(b) subspaces of operating point

(c) stability space

Figure 1. The formation of subspaces.

\subsection{Coordinated Design of PSSs}

Power system stabilizers (PSSs) have been an effective and economic way to dampen power system low-frequency oscillations for decades. In each operating subspace, multiple PSSs are designed in coordination to ensure the power system operating point to be within the stable region [1]. The participators are used to determine the locations of PSSs to be tuned to achieve effective damping result. The generator speed and electromagnetic power are the input signals of PSS2A as shown in Figure 2. To save the space, details of coordinated design are not presented here. Principle of design is given in reference [17]. $\Delta \omega$ is the generator speed deviation, $\Delta P$ is the electromagnetic power deviation. $T_{w 1}, T_{w 2}, T_{w 3}, T_{w 4}$ are the time constants of washout blocks. $T_{1}, T_{3}, T_{8}$ are the lead time constants of phase compensation blocks. $T_{2}, T_{4}, T_{9}$ are the lag time constants of phase compensation 
blocks. $N$ is the number of low-pass filters. $T_{6}, T_{7}$ are the time constants of first-order inertia blocks. $K_{s 1}, K_{s 2}, K_{s 3}$ are the gains of PSS.

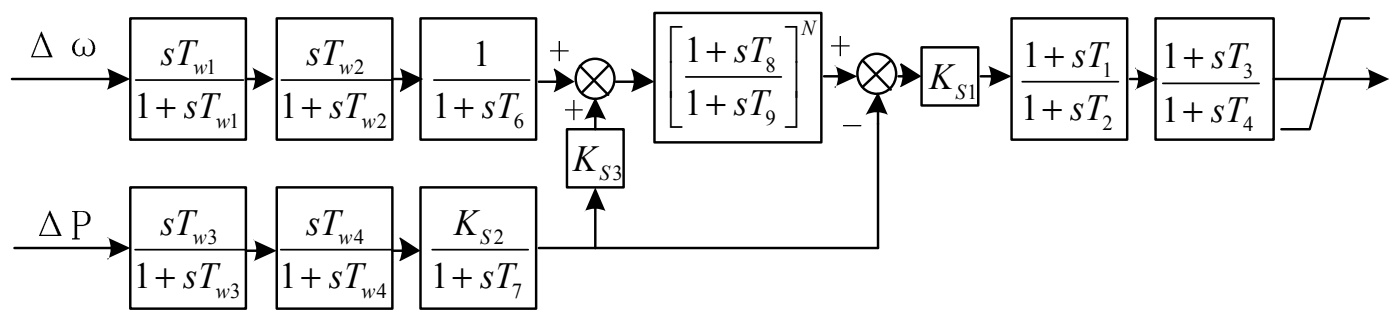

Figure 2. Structure of PSS2A.

\subsection{The CART-Based Adaptive Damping Control Scheme}

CART is a recursive partitioning method which builds classification and regression trees. Classification trees are built for obtaining the splitting rules of different operating point subspaces whereas regression trees are built for identifying which subspace of an operating point the power system is in. In this way, the coordinated PSSs pre-designed off-line can be switched adaptively using on-line measurements. As long as the wind power output fluctuates, the operating point of the power system will deviate from the initial subspace and randomly move forward to other operating point subspaces or back to the initial subspace. The CART will function to identify the attraction subspace in order for the PSSs to be switched on line.

The CART is structured from the top to the bottom consisting of root node, test nodes and terminal nodes. Each test node or root node corresponds to an optimal splitting rule and a subset of the learning dataset. A terminal node is a pure node which could not be split further. The learning dataset itself corresponds to the root node of the CART. The classification process starts from the top root node, and at each level the subsets will be divided according to the optimal splitting rules. The optimal rules are in the form of "if-then-else" rules. In this paper, each terminal node represents an operating point subspace. The measurements from all operating point subspaces consist of the learning dataset which are the input data of the CART. Reference [18] gives a full introduction of general theory and methods of the CART.

To ensure the accuracy of classification, those measurable and controllable measurements are used as the training data which could characterize the subspaces. Since generators' speed includes the information of power flow routing, topology and the power oscillation modals in a power system, the CART uses the speeds of generators as the learning dataset. However, in most cases, the speeds of generators are not measurable or they are measurable without time tags. Therefore, the generator bus frequencies are employed as the learning dataset in this paper instead of generators' speed. The reason is that the generator bus frequencies, which are the derivation of generators' external bus angle, is an approximation to the generators' speed and the generator bus frequencies can be obtained directly from PMUs.

In a large scale power system, only one measurement could not adequately characterize an operating point subspace. Thus multiple measurements need to be employed for tracking the variation of the power system operating point. Multiple measurements from multiple subspaces make the classification process complex. Therefore, in order to distinguish the features of measurements from different subspaces, the Euclidean distance to the hyperplanes is used as the classification algorithm to process large amounts of measurement data.

Take Figure 3 as an example. The small circles and stars represent the measurements from subspaces $\alpha$ and $\beta$, respectively. The horizontal ordinate and vertical ordinate denote the measurements 1 and 2, respectively. Then the dataset from two measurements is expressed in a two dimensional space. In the same way, an $\mathrm{n}$-dimension space is needed when there are $\mathrm{n}$ measurements. As shown in Figure 3, a line can distinguish two groups of data and obviously a plane is needed for a three dimensional space. In the large scale power system, with multiple measurements, a hyper-plane is 
used in this paper to distinguish the subspaces of operating points. In order to explain the algorithm developed in this paper, an example with two measurements is deduced as follows.

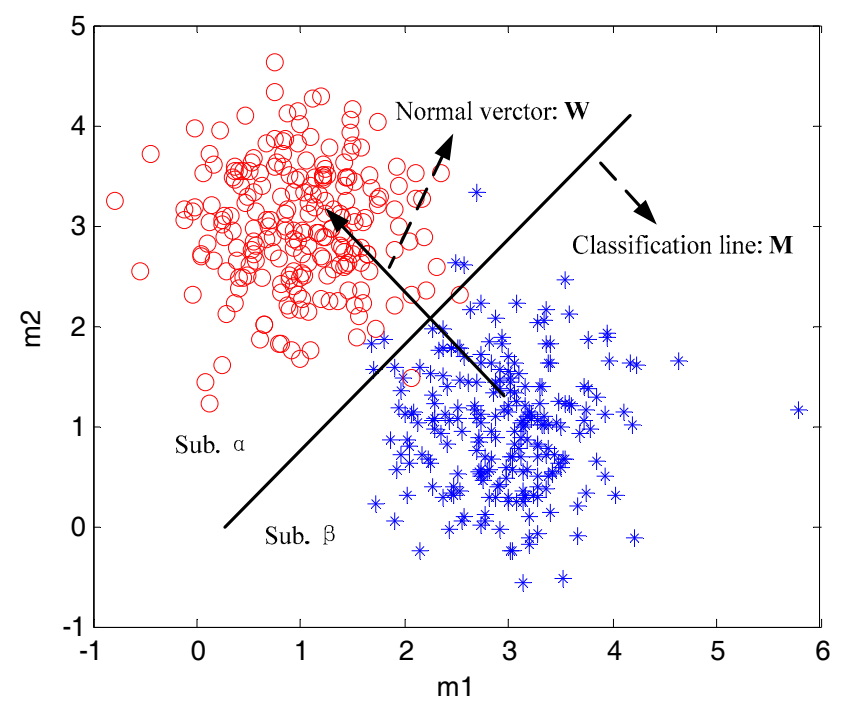

Figure 3. The optimal classification line to classify two groups of data in two dimension space.

Assume a sample from $\alpha$ group has the coordinates value $\left(x_{\alpha i}, y_{\alpha i}\right)$, where $x_{\alpha i}$ and $y_{\alpha i}$ represent the values of $x$-axis and $y$-axis of the $i$-th sample. The values of $x$-axis and $y$-axis of the $j$-th sample from group $\beta$ are denoted $\left(x_{\beta j}, y_{\beta j}\right)$. Then the means of samples from $\alpha$ group could be obtained by:

$$
\boldsymbol{\mu}_{\alpha}=\left(\sum_{i=1}^{n} x_{\alpha i}, \sum_{i=1}^{n} y_{\alpha i}\right) / n
$$

where $n$ represents the number of samples from the $\alpha$ group. The means of samples from the $\beta$ group could be obtained by:

$$
\boldsymbol{\mu}_{\beta}=\left(\sum_{j=1}^{m} x_{\beta j}, \sum_{j=1}^{m} y_{\beta j}\right) / m
$$

where $m$ represent the number of samples from the $\beta$ group. Denote $\boldsymbol{\Sigma}_{\alpha}$ and $\boldsymbol{\Sigma}_{\beta}$ the covariance of measurements of subspaces $\alpha$ and $\beta$, respectively. There should be many lines between those two groups of data. $\mathbf{M}$ denotes the classification line, and $\mathbf{W}$ denotes the normal vector of $\mathbf{M}$. The classification rules of two classes with different covariance is defined as the ratio of the variance between the classes to the variance within the classes, which is named as FLD index [19]. Mathematically, it is:

$$
S=\frac{\left(\mathbf{W}^{\mathrm{T}} \boldsymbol{\mu}_{\alpha}-\mathbf{W}^{\mathrm{T}} \boldsymbol{\mu}_{\beta}\right)^{2}}{\mathbf{W}^{\mathrm{T}} \boldsymbol{\Sigma}_{\alpha} \mathbf{W}+\mathbf{W}^{\mathrm{T}} \boldsymbol{\Sigma}_{\beta} \mathbf{W}^{\prime}}
$$

The FLD index is the best for discriminating two groups of data when the FLD index $S$ is greatest. To achieve maximum value of $S$, the normal vector $\mathbf{W}$ is found to be given by:

$$
\mathbf{W}=\frac{\boldsymbol{\mu}_{\alpha}-\boldsymbol{\mu}_{\beta}}{\boldsymbol{\Sigma}_{\alpha}+\boldsymbol{\Sigma}_{\beta}}
$$

The middle point $\mathbf{C}_{\text {mid }}=\frac{1}{2}\left(\boldsymbol{\mu}_{\alpha}+\boldsymbol{\mu}_{\beta}\right)$ should be on this line. With the point on the line and the normal vector $\mathbf{W}$ identified, the best line is determined. Then the following step is to find the distance from a point $(x, y)$ in the two-dimensional space to the classification line $\mathbf{M}$ as shown below: 


$$
d(x, y)=\frac{a x+b y+c}{\sqrt{a^{2}+b^{2}}}
$$

where the classification line is $\mathbf{M}: a x+b y+c=0$. In this way, a single dimensional variable (distance) $d(x, y)$ is used as the training data instead of two dimensional data. If $d \geq 0$, the operating point is identified to be inside subspace $\alpha$, otherwise the point is inside subspace $\beta$.

Extend the example above in two-dimension space to the case in a multiple-dimension space. It is easy to understand that the hyper-plane can be used to classify subspaces in the high-dimension space as illustrated as follows.

In an n-dimension space, the normal vector of a hyper-plane can also be calculated by (3). The vector composed of middle points between the means is also on the hyper-plane $\mathbf{N}$ :

$$
\mathbf{N}: \mathbf{W} \cdot\left(\boldsymbol{\gamma}-\mathbf{C}_{\text {mid }}\right)=0,
$$

where $\gamma$ represents the position of a point in $n$-dimension space, i.e., $\gamma=(x, y, z, \cdots)$. The distance from a point $\gamma_{i}=\left(x_{i}, y_{i}, z_{i}, \cdots\right)$ to the hyper-plane can be obtained according to (5):

$$
d_{i}=\frac{\mathbf{W} \cdot\left(\gamma_{i}-\mathbf{C}_{m i d}\right)}{\|\mathbf{W}\|}
$$

In this way, the distance vectors composed of the distance from the points of the subspaces to the hyper-planes are identified. Then they are used as the input variables for the CART to perform the classification process and achieve the splitting rules. After the process of classification, the regression process is performed to identify which subspace the current operating point is in. A new distance vector $d^{\prime}$ from current operating point $\gamma^{\prime}$ to the hyper-planes is obtained and used as inputs to the CART, thus the terminal node which characterizes a subspace is reached. In this way, the CART can track the variation of the system operating point and thus guide the updating of appropriate PSSs into service adaptively.

\subsection{Design Procedure of Adaptive Control Scheme}

The design process of the adaptive control scheme presented in this paper includes two stages as shown in Figure 4. The first stage is off-line. Firstly, the power system operating space is divided into different operating subspaces according to the partition of the partition of stochastically variable wind power output. Then the mapping rules are set up and the coordinated multiple PSSs are predesigned for each subspace of power system operating points according to the mapping rules, on the other hand, the model of hyper-planes for classifying subspaces are built, thus the optimum CART and the splitting rules. The second stage is on-line, as shown in Figure 5. The wide-area information from PMUs is collected and the suitable bus frequencies are chosen as the original learning dataset. Then, the distance vector to the hyper-planes are calculated for the inputs of CART for regression. The splitting rules are applied for a regression tree and finally the terminal node could demonstrate the operating subspace, thus the switch order according to the output of the CART and the appropriated PSSs are put into service for damping. Hence in the adaptive damping control scheme, the CART plays a centralized role of decision maker and the multiple PSSs play the role of actuators.

The process of computing the parameters of designed PSS is done off-line, and as a matter of fact, the total duration of the proposed method only include the time for collecting data from PMUs, communication lag time, time of classification and regression caused by CART. The data collection from PMUs could be neglected as it is predesigned beforehand. The delays of WAMS communication are less than several hundred milliseconds. The time of classification and regression caused by CART could also be neglected without the consideration of the commutating ability of the computers. Therefore, the total duration of the on-line action is only composed of the communication lag time, so it is could be fast enough as an online method in a power system. In China, the online decision maker has been 
applied to the South China power grid using the modulation signals to be sent remotely to the DC links and it works well for damping the oscillations [20,21].

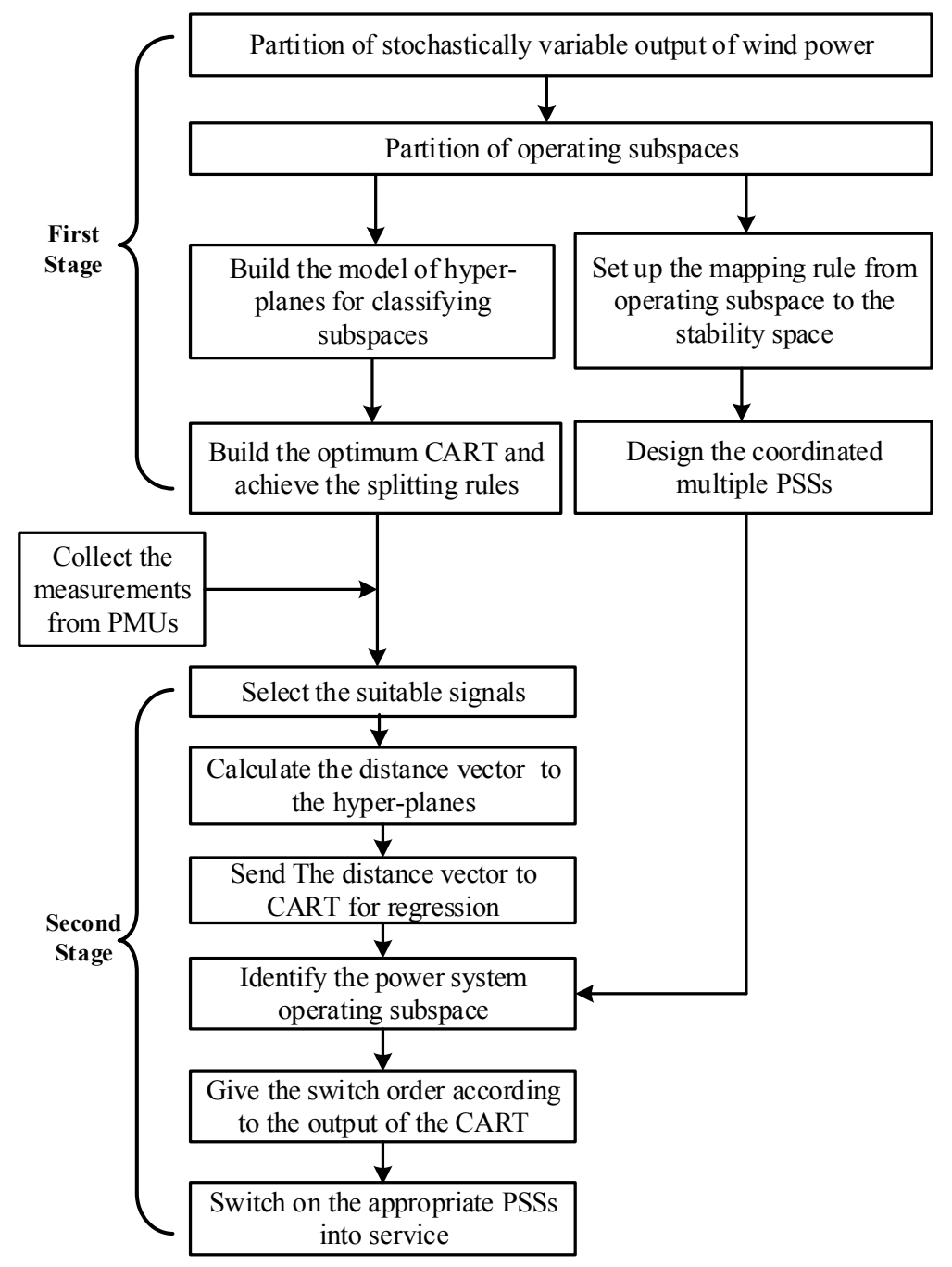

Figure 4. The flowchart of the design process.

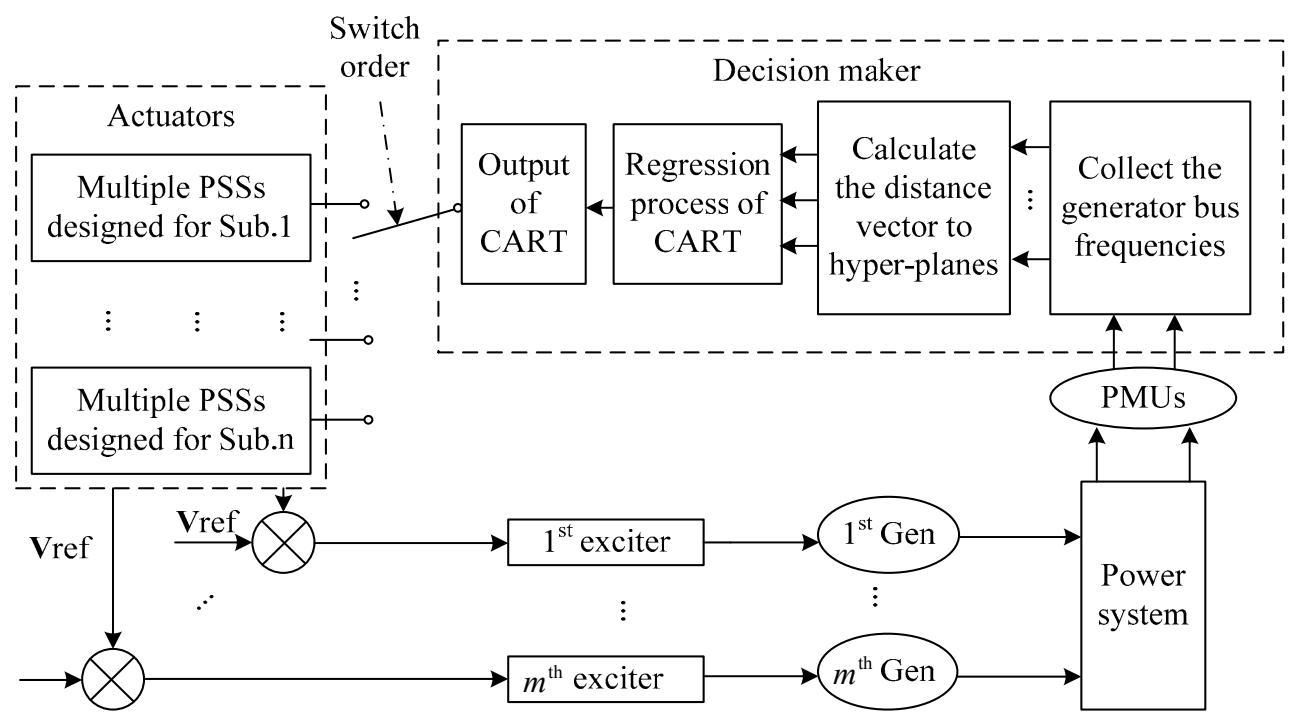

Figure 5. Configuration of the proposed adaptive damping control scheme. 


\section{Results and Analysis}

\subsection{Test System}

A modified 16 generator, 68 bus system [17] with an integrated wind farm is employed for testing the performance of the proposed adaptive damping control scheme. The configuration of the power system is shown in Figure 6. The wind farm is assumed to be at bus 69. The wind farm is modeled as a dynamic equivalence of doubly-fed wind farms using the method shown in reference [22]. In China, at present the most dominant wind turbine generators are doubly fed Induction Generator (DFIG) units, therefore, in this paper, the dynamic equivalence model of doubly-fed wind farms is applied.

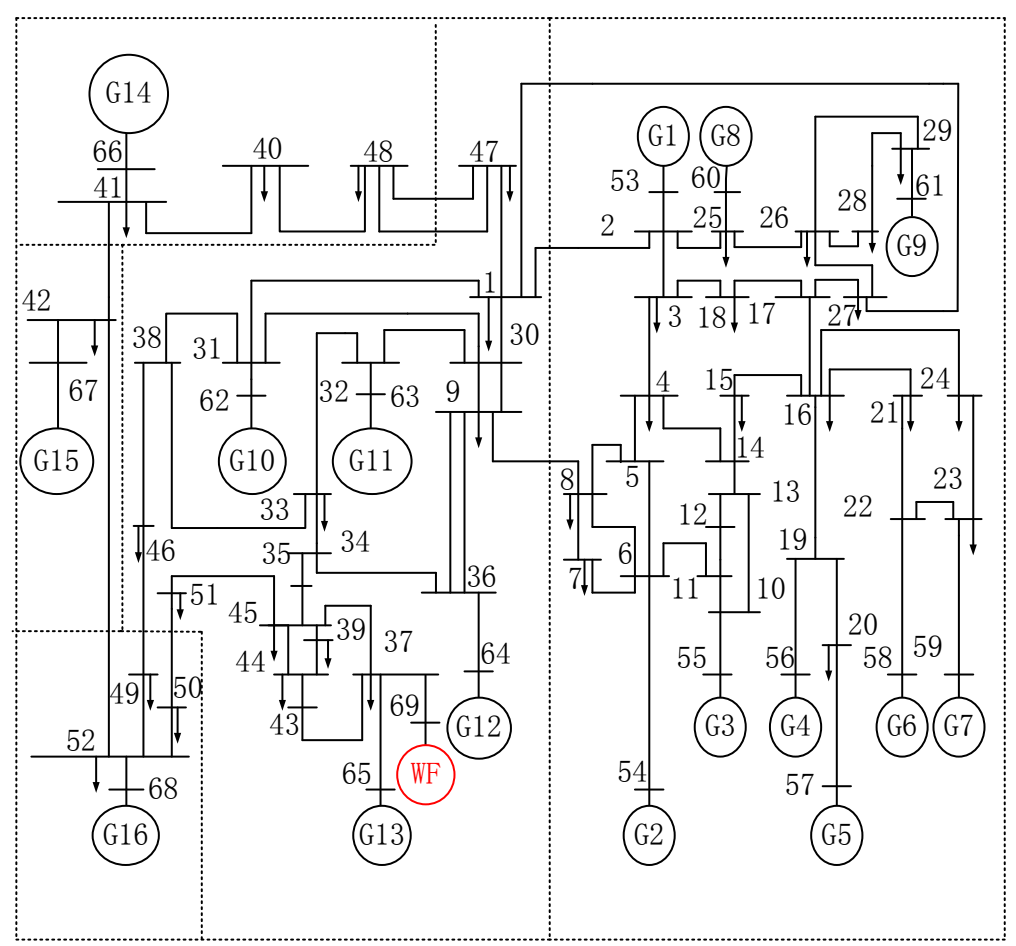

Figure 6. The modified of 16-machine 68-bus system.

The frequency and damping ratio of those modes are given in Figure 7 when the wind power is variable. The stochastically variable output of wind power is divided into 10 subspaces. Each subspace characterizes an operating subspace. The minimum and maximum output of wind power is $0 \mathrm{MW}$ and $4048 \mathrm{MW}$, respectively. In China, the maximum capacity of the Jiuquan wind power base in Gansu Province is up to 5409.2 MW with a penetration of about 22.3\% in 2014 [23]. The large scale of wind power generation has grown rapidly, and ultra-high voltage transmission lines are built for transferring this renewable resource generation powers from the northwest part of the country to the southeast part. Therefore, it is reasonable to consider a large wind farm with high capacity connected to one bus. From Figure 7 it can be seen that with the changing wind power penetration, the damping ratios of different modes changed in a different way. The stochastic fluctuation of wind power affects the damping of system low-frequency power oscillation and can lead to instability in the worst case. 

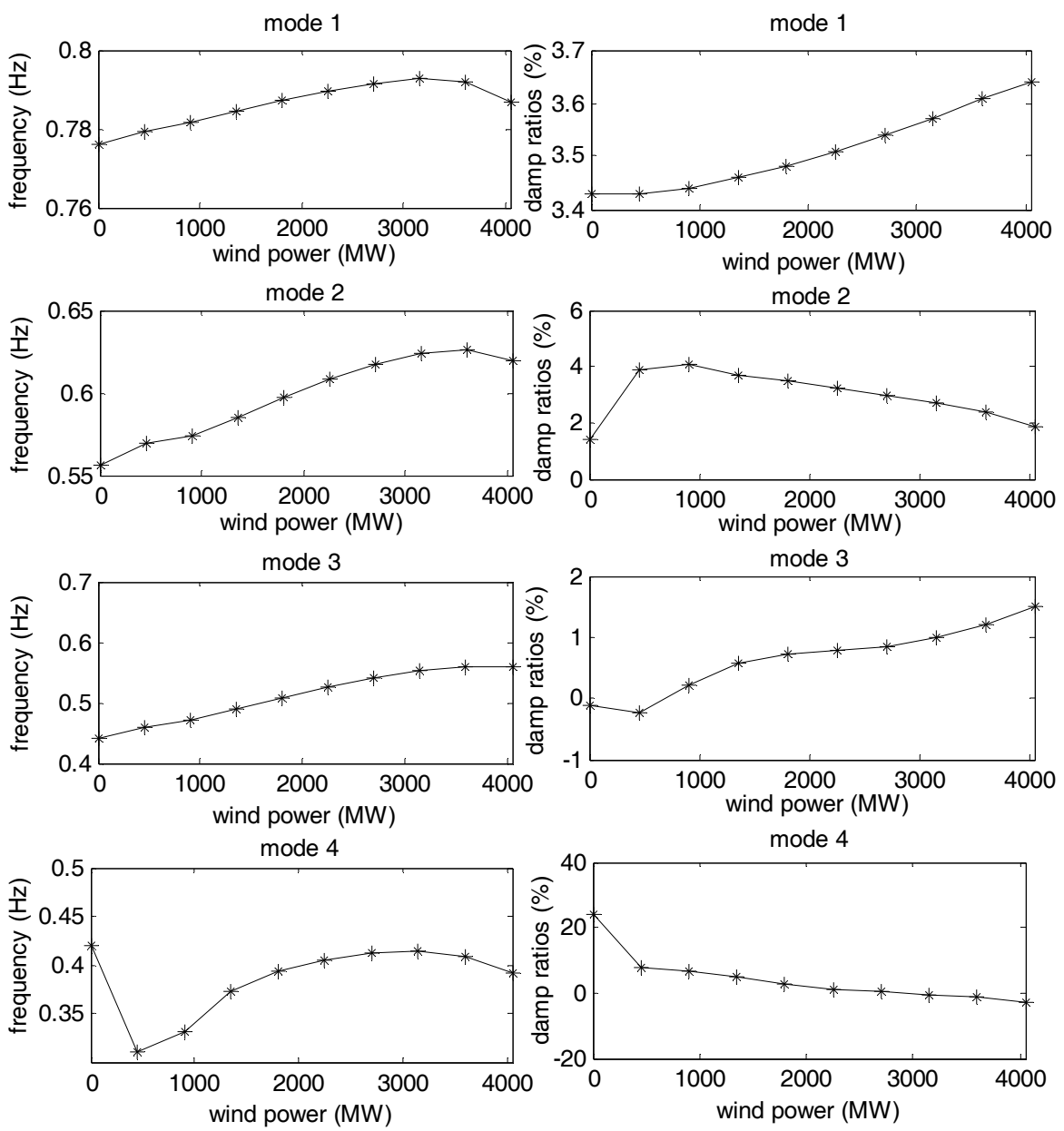

Figure 7. Variation of frequency and damp ratio of oscillation modes with wind power output.

Each generator is installed with a PSS. For each of 10 subspaces of the system operating point, a group of PSSs is selected by use of participation factors and the parameters of the group of PSSs are set in coordination to provide sufficient damping to four oscillation modes. The coordinated PSSs are designed by the traditional method, which includes phase compensator tuning and gain tuning. The compensator tuning is used to provide the required phase compensation for the phase lag between the exciter reference point and the generator air-gap torque. The tuning of an appropriate PSS gain is to ensure that the maximum possible damping can be provided by the PSS for the target modes while limiting the side effect within the acceptable level. The variation from wind power has an impact to the oscillation modes, thus only one set of coordinated PSSs could not provide enough damping for all the subspaces. Therefore, many set of PSSs are designed for many subspaces. The parameters of PSSs designed for one subspace are different from other subspaces. There are 10 subspaces in this paper, thus 10 sets of PSSs. The PSSs combination of each subspace is different from each other as each PSSs combination is designed at its own subspace, respectively.

Once the wind power output changes, the PSSs are switched from one set to another with the guidance of CART. Meanwhile, as long as the parameters of PSSs are coordinated well, a suitable set of parameters of PSSs could provide enough damping for subspaces even if the wind power impact is considered. The results of off-line coordinated design of PSSs are presented in Table 1, and the off-line coordinated design of PSSs is effective and different set of coordinated PSSs could provide satisfying damping to its own subspace.

The system space is divided into $n$ subspaces according to two constraints. The first one is that predesigned PSSs in each subspace could give good performance on the boundary of each subspace to guarantee the subspaces could cover the whole system space. The second one is that CART could 
classify the system space with high accuracy and less complexity to avoid that too large number of subspaces causes the computation burden.

Table 1. Damping ratio and frequency of inter-area modes with PSSs.

\begin{tabular}{|c|c|c|c|c|c|c|}
\hline Subspace & Wind Power Outputs & Modes & 1 & 2 & 3 & 4 \\
\hline \multirow{2}{*}{1} & \multirow{2}{*}{0} & $f(\mathrm{~Hz})$ & 0.6752 & 0.6414 & 0.5621 & 0.3482 \\
\hline & & damp (\%) & 7.39 & 14.04 & 11.95 & 24.73 \\
\hline \multirow{2}{*}{2} & \multirow{2}{*}{450} & $f(\mathrm{~Hz})$ & 0.6973 & 0.6463 & 0.5746 & 0.3839 \\
\hline & & damp (\%) & 10.81 & 13.47 & 12.94 & 22.6 \\
\hline \multirow{2}{*}{3} & \multirow{2}{*}{900} & $f(\mathrm{~Hz})$ & 0.6917 & 0.6505 & 0.5895 & 0.4046 \\
\hline & & damp (\%) & 14.09 & 12.98 & 15.14 & 21.85 \\
\hline \multirow{2}{*}{4} & \multirow{2}{*}{1350} & $f(\mathrm{~Hz})$ & 0.8942 & 0.6687 & 0.6175 & 0.5589 \\
\hline & & damp (\%) & 6.55 & 16.13 & 10.08 & 40.78 \\
\hline \multirow{2}{*}{5} & \multirow{2}{*}{1800} & $f(\mathrm{~Hz})$ & 0.8441 & 0.5556 & 0.5513 & 0.4962 \\
\hline & & damp $(\%)$ & 6.35 & 9.13 & 32.6 & 19.94 \\
\hline \multirow{2}{*}{6} & \multirow{2}{*}{2250} & $f(\mathrm{~Hz})$ & 0.8455 & 0.5914 & 0.5811 & 0.5631 \\
\hline & & damp (\%) & 6.56 & 15.89 & 27.81 & 10.08 \\
\hline \multirow{2}{*}{7} & \multirow{2}{*}{2700} & $f(\mathrm{~Hz})$ & 0.8469 & 0.6016 & 0.5727 & 0.5400 \\
\hline & & damp (\%) & 6.27 & 19.28 & 10.06 & 55.07 \\
\hline \multirow{2}{*}{8} & \multirow{2}{*}{3150} & $\mathrm{f}(\mathrm{Hz})$ & 0.8473 & 0.6255 & 0.5771 & 0.5177 \\
\hline & & damp (\%) & 6.45 & 17.92 & 10.14 & 58.6 \\
\hline \multirow{2}{*}{9} & \multirow{2}{*}{3600} & $\mathrm{f}(\mathrm{Hz})$ & 0.6511 & 0.6048 & 0.5452 & 0.3948 \\
\hline & & $\operatorname{damp}(\%)$ & 11.43 & 1023 & 18.7 & 23.18 \\
\hline \multirow{2}{*}{10} & \multirow{2}{*}{4048} & $\mathrm{f}(\mathrm{Hz})$ & 0.8572 & 0.6258 & 0.5738 & 0.4817 \\
\hline & & damp (\%) & 6.27 & 14.02 & 10.03 & 24.3 \\
\hline
\end{tabular}

The interval of subspaces are [0 0.5h], [0.5h 1.5h], [1.5h 2.5h], [2.5h 3.5h], [3.5h 4.5h], [4.5h 5.5h], [5.5h 6.5h], [6.5h 7.5h], [7.5h 8.5h], [8.5h 4048], in which $h=450$. The boundaries of subspaces are 0 , $0.5 h, 1.5 h, \ldots, 4048$. The lower boundary of one subspace is the upper boundary of other subspace. For example, the 2 nd boundary $0.5 \mathrm{~h}$ is the upper boundary in the first subspace and lower boundary of the second subspace. In this way, the whole system space could be covered by subspaces. The lower boundary of the first subspace is 0 as it is the minimum output of wind power. The upper boundary of 10th subspace is 4048 as it is the maximum output of wind power. The coordinated PSSs of each subspace are designed at the center point of each subspace, e.g., $0, h=450,2 h=900,3 h=1350, \ldots$, while the PSSs of the first and last subspace are designed at the points of minimum and maximum of wind power outputs, respectively.

Nine boundary points are used as test cases to demonstrate the robustness of coordinated PSSs and the simulation results are shown in Figure 8. Each sub-plot represents one boundary point. Take the second boundary as shown in Figure 8a as an example, the 2nd boundary point is the upper and lower boundaries of subspaces 1 and 2, respectively. As a matter of fact, this boundary point is in both subspace 1 and subspace 2 . The dashed curves represent the dynamic response without PSSs.

The blue and red curves represent the dynamic responses with different set of coordinated PSSs, respectively. The blue curve represents the response with the first set of coordinated PSSs applied which is designed for subspace 1. The red curve represents the response with the second set of coordinated PSSs applied which is designed for subspace 2. From this figure, it can be seen that both set of PSSs could give good performance which demonstrate the coordinated PSSs designed at the center point of subspace could provide enough damping at the boundaries points which means the classified subspaces could be able to cover the whole system space. From other sub-plots, the simulation results at other boundary points also demonstrate the robustness of the coordinated PSSs. In this way, different set of PSSs could be switched from one to another adaptively without unsatisfactory control performance. 

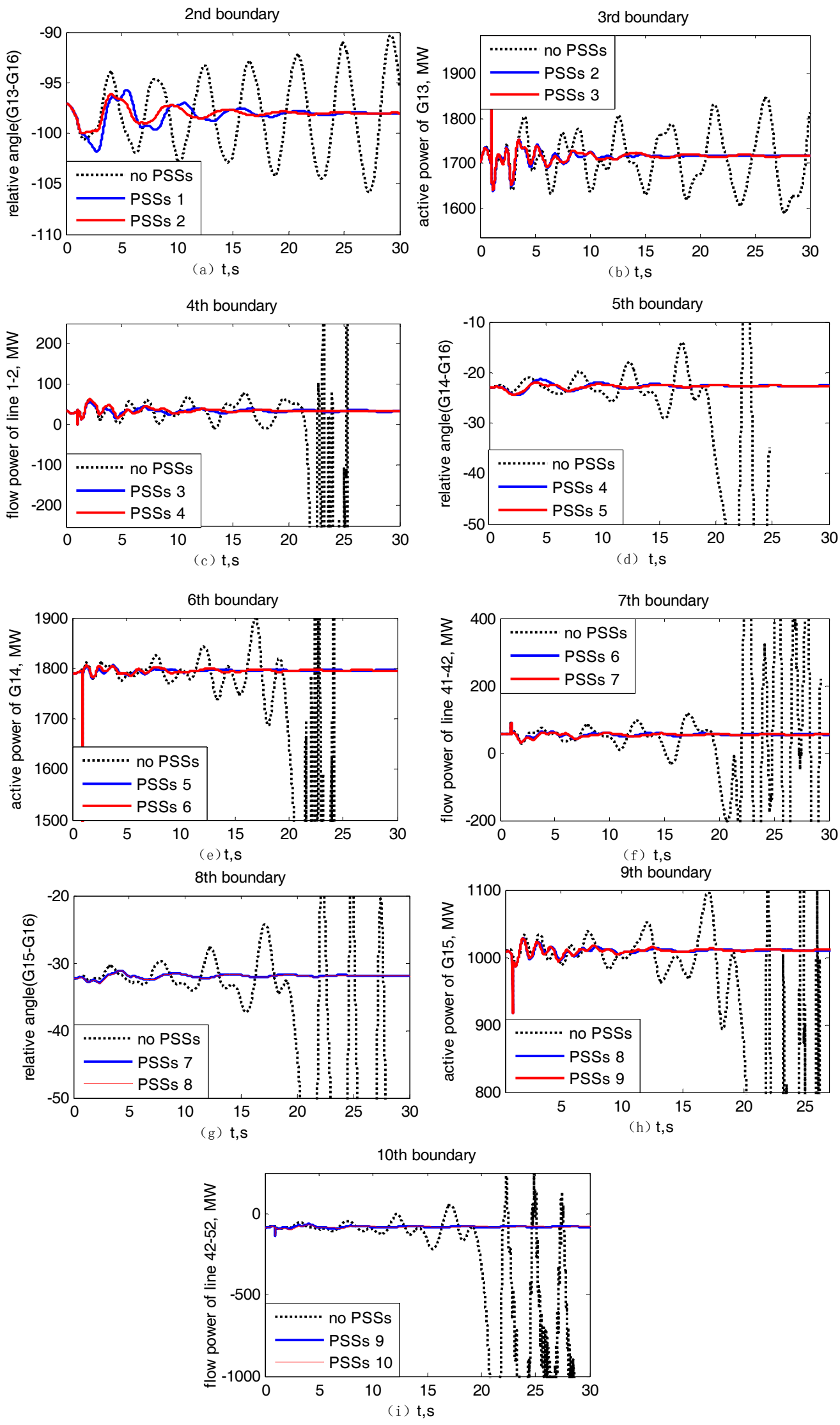

Figure 8. Dynamic responses with different controllers in the case of subspaces boundaries. 
It can be seen from Figure 8 that the inter-area power oscillations can be suppressed effectively with the pre-designed multiple PSSs being matched to the correct subspace of system operating point even when the wind generation varies. Hence PSSs pre-designed need to be adaptively adjusted to following the wind power variation. Otherwise poorly damped inter-area oscillations are observed. An adaptive damping control robust to the changes of power system operating point caused by variation of grid-connected wind power need to be able to track the variation of system operating point and to assign correct group of pre-designed PSSs into the service to suppress system inter-area power oscillations. Function of tracking system operating point when wind generation varies is implemented by the CART formed for the test system.

\subsection{Formation of the CART}

All 10 subspaces are considered to measure the participation factor of each generator. The participation factors with wind power variation are calculated and the results are shown in Table 2. Two highest participation factors of each mode of each subspace are displayed in Table 2. It can be observed that, the participation factors changed with the wind power variation, but they do not change a lot. The measurement used for training the CART is selected according to the highest participation factors of four inter-area oscillations. Hence, measurement of bus frequencies of machine G9, G13, G14 and G15 is used to form the CART.

Table 2. Participation factors of different generators in different subspaces.

\begin{tabular}{cccccccccc}
\hline \multirow{2}{*}{ Sub. No. } & \multicolumn{2}{c}{ Mode 1 } & \multicolumn{2}{c}{ Mode 2 } & \multicolumn{2}{c}{ Mode 3 } & \multicolumn{2}{c}{ Mode 4 } \\
\cline { 2 - 9 } & Gen. No. & PF & Gen. No. & PF & Gen. No. & PF & Gen. No. & PF \\
\hline \multirow{2}{*}{ Sub. 1 } & 15 & 1.00 & 13 & 1.00 & 14 & 1.00 & 13 & 1.00 \\
& 14 & 0.37 & 9 & 0.19 & 16 & 0.49 & 9 & 0.28 \\
\hline \multirow{2}{*}{ Sub. 2 } & 15 & 1.00 & 13 & 1.00 & 14 & 1.00 & 13 & 1.00 \\
& 14 & 0.38 & 9 & 0.20 & 16 & 0.68 & 14 & 0.19 \\
\hline \multirow{2}{*}{ Sub. 3 } & 15 & 1.00 & 13 & 1.00 & 14 & 1.00 & 13 & 1.00 \\
& 14 & 0.38 & 9 & 0.27 & 16 & 0.82 & 16 & 0.21 \\
\hline \multirow{2}{*}{ Sub. 4 } & 15 & 1.00 & 13 & 1.00 & 16 & 1.00 & 13 & 1.00 \\
& 14 & 0.39 & 9 & 0.29 & 14 & 0.97 & 16 & 0.23 \\
\hline \multirow{2}{*}{ Sub. 5 } & 15 & 1.00 & 13 & 1.00 & 16 & 1.00 & 13 & 1.00 \\
& 14 & 0.39 & 9 & 0.29 & 14 & 0.78 & 15 & 0.38 \\
\hline \multirow{2}{*}{ Sub. 6 } & 15 & 1.00 & 13 & 1.00 & 16 & 1.00 & 13 & 1.00 \\
& 14 & 0.39 & 9 & 0.28 & 14 & 0.67 & 15 & 0.61 \\
\hline \multirow{2}{*}{ Sub. 7 } & 15 & 1.00 & 13 & 1.00 & 16 & 1.00 & 13 & 1.00 \\
& 14 & 0.39 & 9 & 0.27 & 14 & 0.61 & 14 & 0.87 \\
\hline \multirow{2}{*}{ Sub. 8 } & 15 & 1.00 & 13 & 1.00 & 16 & 1.00 & 14 & 1.00 \\
& 14 & 0.39 & 9 & 0.28 & 14 & 0.58 & 15 & 0.95 \\
\hline \multirow{2}{*}{ Sub. 9 } & 15 & 1.00 & 13 & 1.00 & 16 & 1.00 & 14 & 1.00 \\
& 14 & 0.39 & 9 & 0.29 & 14 & 0.59 & 15 & 1.00 \\
\hline \multirow{2}{*}{ Sub. 10 } & 15 & 1.00 & 13 & 1.00 & 16 & 1.00 & 15 & 1.00 \\
& 14 & 0.40 & 9 & 0.35 & 14 & 0.62 & 14 & 0.68 \\
\hline \multirow{2}{*}{ (15 } & & & & & & & &
\end{tabular}

The sample rate of each measurement is 30 samples per second. For each subspace of system operating point, 100 initial sampled data with 5\% noise are collected. Thus 1000 trajectories for 10 subspaces are employed to form the initial learning dataset of the CART. The initial learning data is a matrix with 1000 rows and 120 columns. 1000 rows are the product of 100 initial sampled data and 10 subspaces. 120 columns are the product of 30 samples and four measurements. Therefore, a hyper-plane of classification is formed in a 120-dimensional space. There are 45 hyper-planes to classify two subspaces. As this is a case of high-dimensional data set. Formation of the CART 
needs to consider the compromise between the accuracy and tree complexity. A small-size CART cannot capture all the dynamic behavior and a large-size CART would bring about the incorrect identification due to the over-fitting [24]. A plot of the misclassification rate for each sub-tree is shown in Figure 9, from which it can be seen that the optimum number of the decision tree is 10 for 10 subspaces. The minimal mismatching rate of the CART is approximately 0.0433 which indicates the $95.67 \%$ probability of correct subspace matching.

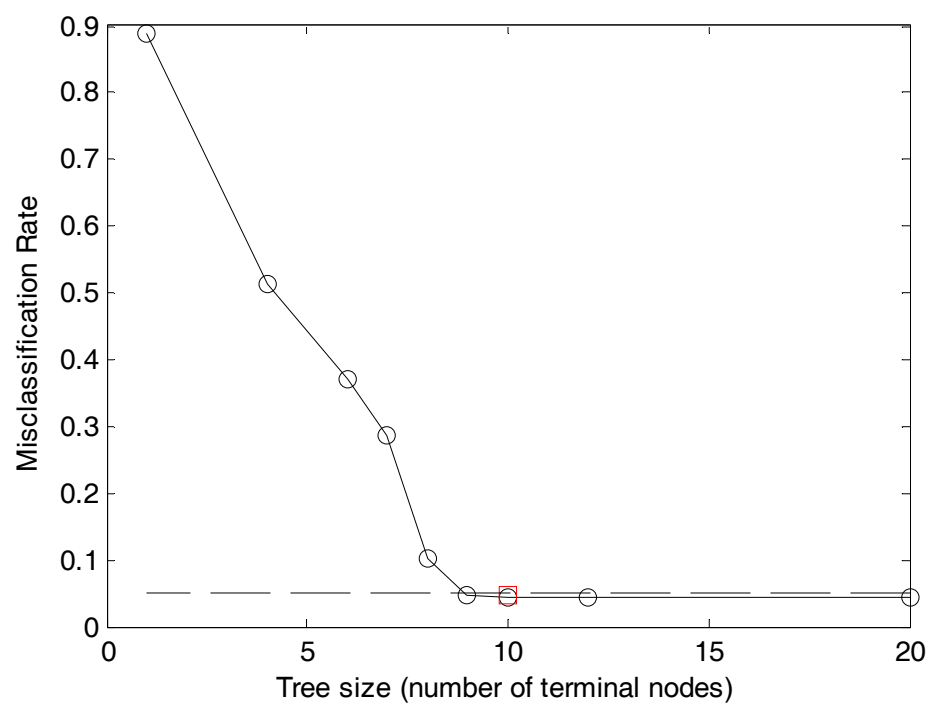

Figure 9. Cross-validation estimate of the misclassification rate.

From Figure 9, it can also be seen that 10 subspaces appeared at the positions of terminal nodes and could be classified with high accuracy, which means the number of subspaces is not too big. Since the big number of subspaces make the CART complex and difficult to classify with high accuracy. Therefore, the number of subspaces 10 is a suitable number in this paper.

After the training process, the classification tree is formed as shown in Figure 10, and the splitting rules for each node are generated at the same time. The structure of formed CART for the test system is shown in Figure 10. It has 10 terminal nodes which represent 10 operating subspaces. The rectangle blocks represent the root node and intermediate nodes. The ellipse blocks are the terminal nodes. At each separation point, there is a splitting rule. The inequations are the splitting rules of each intermediate node which are generated by training process. For example, the distance vector is dropped down to the CART. And the splitting rule of the 1st node circled by a red ellipse block is the distance to the 1st hyper-plane. At the root node, the splitting rule is $d_{1}<0.0263$ and $d_{1} \geq 0.0263$. If $d_{1}$ is smaller than 0.0263 then the distance vector will be dropped down to node 2 for further splitting. If $d_{1}$ is bigger than 0.0263 , then the distance vector will be dropped down to Sub. 1 which is a terminal node and the identification process is stopped. In this way, the current system operating point is identified to be in subspace 1 . The distance vector from an operating point to hyper-planes is expressed as $\mathbf{d}=\left[d_{1}, d_{2}, \cdots, d_{45}\right], d_{i}$ represents the distance to the $i$-th hyper-plane. Because there are 10 operating subspaces, the total number of hyperplanes are 45 , which could classify each two subspaces. In this way, the distance vector actually include 45 distance parameters.

After CART training, the regression test process is performed to test the performance of off line of CART. The test results are shown in Table 3. Outputs of wind power are systematically modified to generate 5000 different system operating points. From each subspace, 500 different system operating points are generated. From Table 3, it can be observed that, 4803 points out of 5000 system states are classified to the correct subspace, and 197 cases are classified to the wrong subspaces. The accuracy of regression test is about $98.9 \%$. The misclassified cases are mostly the points around the boundaries. Also, Figure 8 shows that even the boundaries points are classified to be neighbourhood subspaces, 
and the neighbourhood set of PSSs can also provide satisfying damping. Therefore, good performance of CART when it is applied offline and online can be guaranteed.

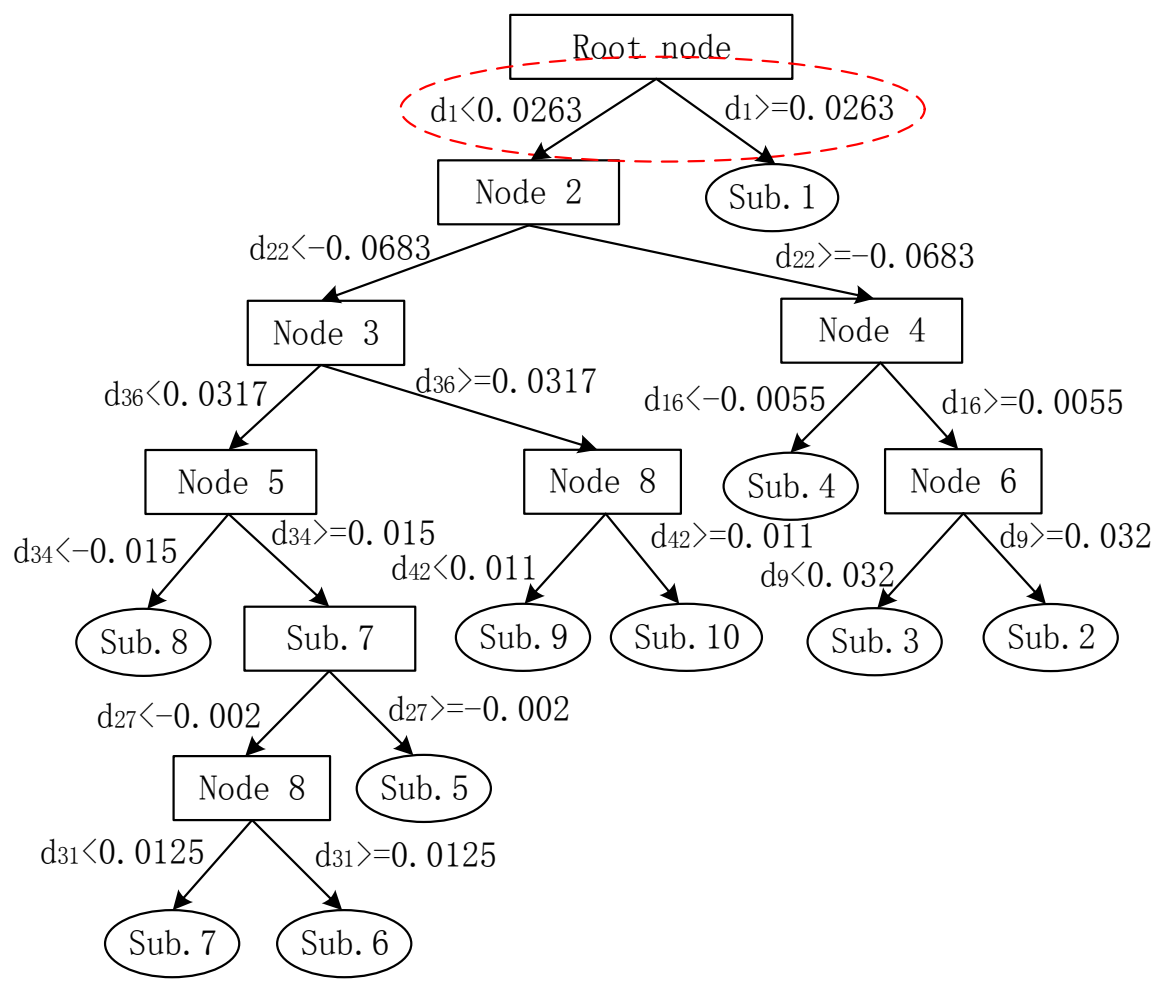

Figure 10. The CART for distinguishing different subspaces of operating point.

Table 3. Cases from subspaces test results.

\begin{tabular}{ccccccccccc}
\hline Substation No. & $\mathbf{1}$ & $\mathbf{2}$ & $\mathbf{3}$ & $\mathbf{4}$ & $\mathbf{5}$ & $\mathbf{6}$ & $\mathbf{7}$ & $\mathbf{8}$ & $\mathbf{9}$ & $\mathbf{1 0}$ \\
\hline Classified Sub.1 & 492 & 10 & 0 & 0 & 0 & 0 & 0 & 0 & 0 & 0 \\
Classified Sub. 2 & 8 & 478 & 8 & 1 & 0 & 0 & 0 & 0 & 0 & 0 \\
Classified Sub. 3 & 0 & 11 & 481 & 10 & 0 & 0 & 0 & 0 & 0 & 0 \\
Classified Sub. 4 & 0 & 1 & 9 & 474 & 15 & 0 & 0 & 0 & 0 & 0 \\
Classified Sub. 5 & 0 & 0 & 0 & 15 & 468 & 15 & 0 & 0 & 0 & 0 \\
Classified Sub. 6 & 0 & 0 & 0 & 0 & 17 & 471 & 12 & 0 & 0 & 0 \\
Classified Sub. 7 & 0 & 0 & 0 & 0 & 0 & 13 & 479 & 13 & 0 & 0 \\
Classified Sub. 8 & 0 & 0 & 0 & 0 & 0 & 1 & 9 & 477 & 6 & 0 \\
Classified Sub. 9 & 0 & 0 & 0 & 0 & 0 & 0 & 0 & 10 & 488 & 5 \\
Classified Sub.10 & 0 & 0 & 0 & 0 & 0 & 0 & 0 & 0 & 5 & 495 \\
\hline
\end{tabular}

\subsection{Simulation Results and Discussions}

The test power system operates initially in subspace 9 with the output of wind power being $3600 \mathrm{MW}$. The simulation runs for $35 \mathrm{~s}$ with wind power output dropped to $1350 \mathrm{MW}$ at $5 \mathrm{~s}$ of simulation due to the disconnection of part of wind power caused by fault. At this time, the wide-area information from PMUs is sent to the CART for identification of variation of system operating point. The outputs from the CART are shown in Figure 11, from which it can be seen that, initially the CART cannot identify the correct system operating subspace. With the increase of number of sampling data, the identification result of the CART converges to the correct system operating subspace. After $t=6 \mathrm{~s}$, the outputs of the CART indicate that the system operates in subspace 4 . Then the multiple PSSs designed for subspace 4 are activated and switched on. 


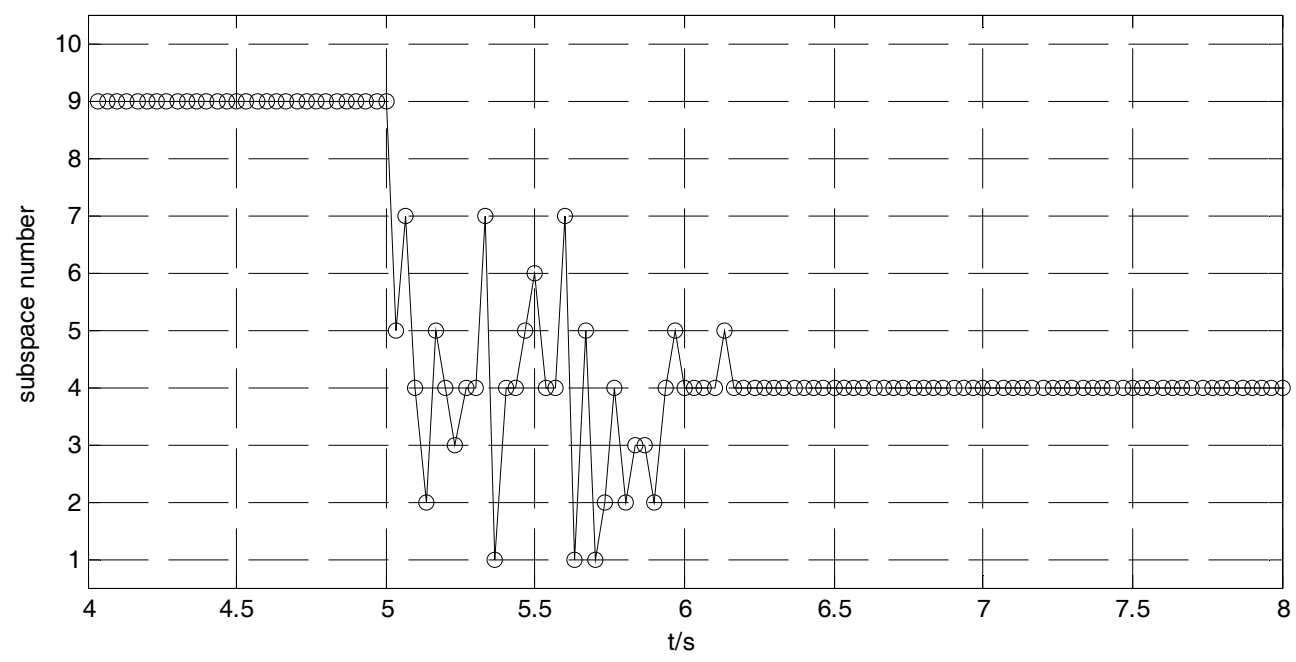

Figure 11. Output trajectory of the CART.

In the simulation part, the wind power output is changed at $t=5 \mathrm{~s}$, and the PSSs are switched at $t=7 \mathrm{~s}$. The time from wind power output changed to the controllers was switched on is about $2 \mathrm{~s}$, which considers communication delay. From Figure 11, the identification process cost about $1 \mathrm{~s}$, the left $1 \mathrm{~s}$ takes the communication delay into account.

Results of dynamic simulation are shown in Figure 12, where the dotted curves are the results with PSSs designed for subspace 9 unchanged (fixed-parameter PSSs). The solid curves are the results with the adaptive PSSs proposed in this paper. It can be seen that the CART based adaptive control scheme can effectively track the variation of power system operating point caused by variable grid-connected wind power, and switch on the appropriate PSSs adaptively to suppress power system inter-area power oscillation.
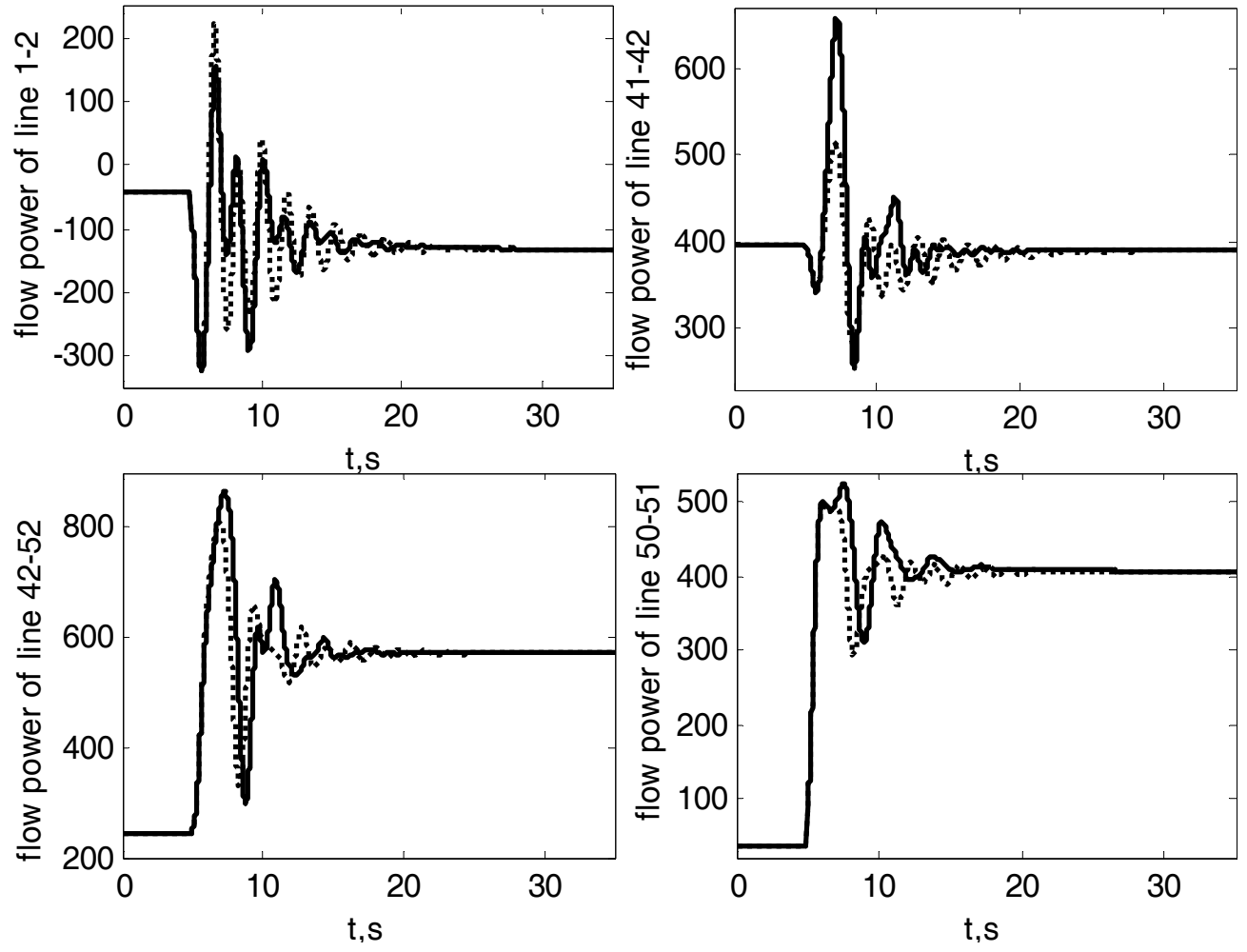

Figure 12. Dynamic responses in case of changing wind power outputs. 
It is worth noting that CART plays a role of mapping the measurement space to state variable space actually. Although the wind power pattern could be different, the state variables could not drift in a dramatic manner. On one hand, from the output of CART as shown in Figure 11, it can be seen that in the initial stage, the output could not converge to a constant number, it needs a while to converge. After about $1 \mathrm{~s}$, the output of CART converge to a constant number. The corresponding set of PSSs are switched on when the output of regression tree converged. On the other hand, in the process of training the CART, the initial sampled data take 5\% noise into count. Therefore, the errors in adjustments could be avoided through modeling the noise to the training dataset.

To further demonstrate the damping improvement, the Prony analysis is performed to analyze the real-time responses and the results are shown in Table 4. Also, the small signal stability analysis is carried out to calculate the oscillation frequency and damping ratios. It can be observed from the Prony analysis and eigenvalue analysis that, the adaptive controllers provide better performance.

Table 4. Prony analysis and eigenanalysis results.

\begin{tabular}{ccccccccc}
\hline & \multicolumn{4}{c}{ Adaptive PSSs } & \multicolumn{4}{c}{ Fixed PSSs } \\
\cline { 2 - 9 } Mode No. & \multicolumn{2}{c}{ Eigenanalysis } & \multicolumn{2}{c}{ Prony Analysis } & \multicolumn{2}{c}{ Eigenanalysis } & \multicolumn{2}{c}{ Prony Analysis } \\
\cline { 2 - 9 } & $f \mathbf{H z})$ & Damp (\%) & $f(\mathbf{H z})$ & Damp (\%) & $f(\mathbf{H z})$ & Damp (\%) & $f(\mathbf{H z})$ & Damp (\%) \\
\hline Mode 1 & 0.5589 & 40.78 & 0.538 & 46.064 & 0.4978 & 30.46 & 0.479 & 20.145 \\
Mode 2 & 0.6175 & 10.08 & 0.617 & 10.412 & 0.5536 & 7.24 & 0.565 & 6.88 \\
Mode 3 & 0.6687 & 16.13 & 0.633 & 17.953 & 0.5986 & 15.20 & 0.614 & 12.989 \\
Mode 4 & 0.8942 & 6.55 & 0.854 & 6.666 & 0.8725 & 5.64 & 0.887 & 5.994 \\
\hline
\end{tabular}

\subsection{Test System with Multiple Wind Farms}

The 16-generator system with three wind farms added at different buses is used as the test system for investigating the effectiveness of the proposed adaptive control. Assume the first wind farm is added at bus 42 , the 2 nd wind farm is connected at bus 42 , and the 3rd wind farm is connected at bus 52 . The operating subspaces are divided with different wind farm outputs as shown in Table 5 . The set of PSSs are predesigned for different subspaces and the new CART are also trained off-line with dataset obtained from this test system. The time domain simulations are carried out in the case of multi wind farms connected to different buses.

Table 5. The Subspaces with different WF outputs.

\begin{tabular}{cccc}
\hline Sub. No. & 1st WF Outputs & 2nd WF Outputs & 3rd WF Outputs \\
\hline 1 & 450 & 450 & 450 \\
2 & 450 & 450 & 900 \\
3 & 450 & 900 & 450 \\
4 & 450 & 900 & 900 \\
5 & 900 & 450 & 450 \\
6 & 900 & 450 & 900 \\
7 & 900 & 900 & 450 \\
8 & 900 & 900 & 900 \\
\hline
\end{tabular}

Assume the power system is operating at the output of three wind farms are all $450 \mathrm{MW}$, which means the power system is operating at the first space. When $t=5 \mathrm{~s}$ the output of $1 \mathrm{st}$ wind farm increased to $900 \mathrm{MW}$. When $t=15 \mathrm{~s}$ the output of 2 nd wind farm increased to $900 \mathrm{MW}$. When $t=25 \mathrm{~s}$ the output of 3rd wind farm increased to 900 MW. The output of CART are shown in Figure 13, from which it can be seen that the initial output of the CART shows the power system is operating in subspace 1 before $t=5 \mathrm{~s}$. After $5 \mathrm{~s}$, the output shows the power system operating subspace changed to subspace 5. After $15 \mathrm{~s}$ the output of CART demonstrate the subspace 7 and then subspace 8 after $25 \mathrm{~s}$. The output trajectory of CART shows that CART could track the operating subspace adaptive to the changing wind power outputs and give the correct switch order. 


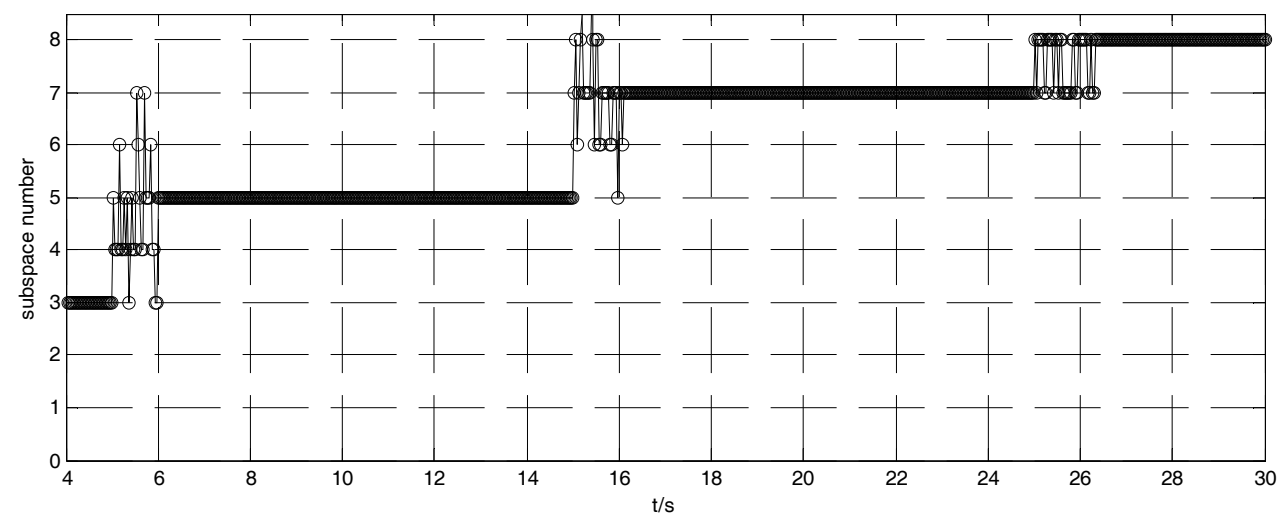

Figure 13. Output trajectory of the CART in the case of multiple wind farms.

The dynamic response of relative angular of generators 1 and 16 is displayed in Figure 14. The dashed curve is the dynamic response of relative angular with the fixed set of PSSs predesigned for subspace 3. The solid curve is the relative angular with the adaptive control method proposed in this paper. From this figure, it can be seen that the adaptive control method show good performance even if the multiple wind farms were connected to different buses.

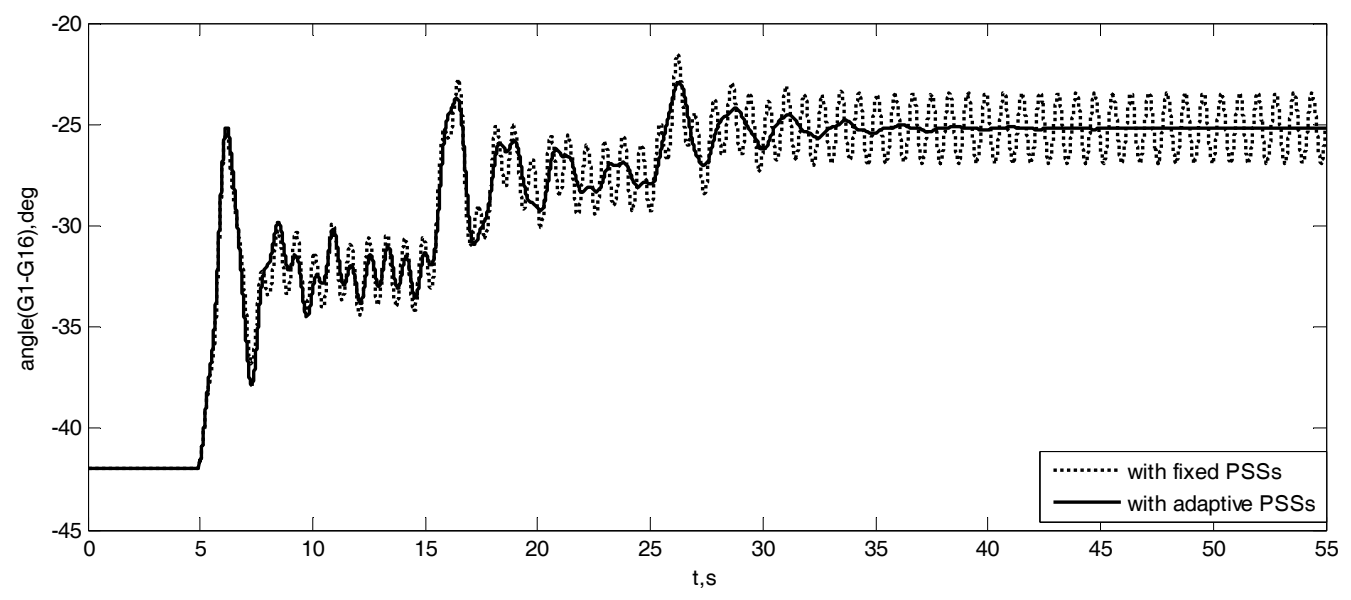

Figure 14. Dynamic responses in case of changing wind power outputs.

\section{Conclusions}

An adaptive damping control robust to stochastic varying operating conditions caused by integrated wind power is proposed in this paper. Firstly, the space of operating point is divided into operating subspaces by the even interval of wind power outputs. Secondly, coordinated PSSs are pre-designed for each operating subspace. Thirdly, a classification tree is formed by training the distances to the hyperplanes, and the regression tree is used for regression to identify the subspaces with the help of on-line measurement from PMUs. In this way, the dynamic behavior of a varying power system is tracked and the appropriate coordinated PSSs are switched into service adaptively. The simulation results of modified test system integrated with wind generation are presented in the paper to demonstrate the robustness of the proposed adaptive damping control based on CART to the variation of system operating point in suppressing system low-frequency power oscillations when grid-connected wind power varies. Furthermore, the proposed adaptive damping control also show the good performance in the case of multiple wind farms connected at different buses. 
Author Contributions: For research articles with several authors, a short paragraph specifying their individual contributions must be provided. The following statements should be used "Conceptualization, J. D. and T.W.; Methodology, J.Y.; Software, J.Y.; Validation, F.C., and S.P.; Formal Analysis, J.D.; Investigation, J.D.; Resources, S.P.; Data Curation, S.P.; Writing-Original Draft Preparation, T.W.; Writing-Review \& Editing, J.Y.; Visualization, J.S.; Supervision, F.C.; Project Administration, T.W.; Funding Acquisition, T.W.", please turn to the CRediT taxonomy for the term explanation. Authorship must be limited to those who have contributed substantially to the work reported.

Funding: This research was funded by Fundamental Research Funds for the Central Universities grant number 2018MS006.

Conflicts of Interest: The authors declare no conflict of interest.

\section{Nomenclature}

$\Delta \omega \quad$ generator speed deviation, p.u.

$\Delta P \quad$ electromagnetic power deviation, p.u.

$T$ time constants of washout blocks, $\mathrm{s}$

$N \quad$ number of low-pass filters

$K \quad$ the gain of PSS

$\alpha \quad$ the measurements from circles

$\beta \quad$ the measurements from stars

$\mu_{\alpha} \quad$ the means of measurements $\alpha$

$\mu_{\beta} \quad$ the means of measurements $\beta$

$\Sigma_{\alpha}$ the covariance of measurements of subspaces $\alpha$

$\Sigma_{\beta}$ the covariance of measurements of subspaces $\beta$

M the classification line

W the normal vector

d the distance vector

$\mathbf{N}$ the direction vector of hyper-plane

$\mathbf{C}_{\text {mid }} \quad$ the middle point

$f \quad$ frequency of oscillation mode, $\mathrm{Hz}$

\section{References}

1. Kunder, P. Power System Stability and Control; McGraw-Hill: New York, NY, USA 1994.

2. Yu, X.; Zhang, W.; Zang, H.; Yang, H. Wind power interval forecasting based on confidence interval optimization. Energies 2018, 11, 3336. [CrossRef]

3. Bai, W.; Lee, D.; Lee, K.Y. Stochastic dynamic AC optimal power flow based on a multivariate short-term wind power scenario forecasting model. Energies 2018, 12, 2138. [CrossRef]

4. Zhao, X.; Lin, Z.; Fu, B.; He, L.; Fang, N. Research on automatic generation control with wind power participation based on predictive optimal 2-degree-of-freedom PID strategy for multi-area interconnected power system. Energies 2018, 11, 3325. [CrossRef]

5. Divshali, P.H.; Choi, B.J.; Liang, H. Multi-agent transactive energy management system considering high levels of renewable energy source and electric vehicles. IET Gener. Transm. Distrib 2017, 11, 3713-3721. [CrossRef]

6. Athari, M.H.; Wang, Z. Impacts of wind power uncertainty on grid vulnerability to cascading overload failures. IEEE Trans. Sustain. Energy 2018, 9, 128-137. [CrossRef]

7. Zhang, J.; Ju, P.; Yu, Y.; Wu, F. Responses and stability of power system under small Gauss type random excitation. Sci. China Technol. Sci. 2012, 55, 1873-1880. [CrossRef]

8. Charles, K.; Urasaki, N.; Senjyu, T.; Elsayed Lotfy, M.; Liu, L. Robust load frequency control schemes in power system using optimized PID and model predictive controllers. Energies 2018, 11, 3070. [CrossRef]

9. Mansiri, K.; Sukchai, S.; Sirisamphanwong, C. Fuzzy control for smart PV-battery system management to stabilize grid voltage of $22 \mathrm{kV}$ distribution system in Thailand. Energies 2018, 11, 1730. [CrossRef]

10. Majumder, R.; Chaudhuri, B.; Pal, B.C. A probabilistic approach to model-based adaptive control for damping of interarea oscillations. IEEE Trans. Power Syst. 2005, 20, 367-374. [CrossRef] 
11. Chaudhuri, B.; Majumder, R.; Pal, B.C. Application of multiple-model adaptive control strategy for robust damping of interarea oscillations in power system. IEEE Trans. Contr. Syst. Technol. 2004, 12, 727-736. [CrossRef]

12. Wang, T.; Pal, A.; Thorp, J.; Yang, Y. Use of polytopic convexity in developing an adaptive interarea oscillation damping scheme. IEEE Trans. Power Syst. 2017, 32, 2509-2520. [CrossRef]

13. Ye, H.; Liu, Y. Design of model predictive controllers for adaptive damping of inter-area oscillations. Int. J. Electr. Power Energy Syst. 2013, 45, 509-518. [CrossRef]

14. Bernabeu, E.E.; Thorp, J.S.; Centeno, V. Methodology for a security/dependability adaptive protection scheme based on data mining. IEEE Trans. Power Syst. 2012, 27, 104-111. [CrossRef]

15. Shi, X.; Lei, X.; Huang, Q.; Huang, S.; Ren, K.; Hu, Y. Hourly day-ahead wind power prediction using the hybrid model of variational model decomposition and long short-term memory. Energies 2018, 11, 3227. [CrossRef]

16. Cai, K.; Alalibo, B.P.; Cao, W.; Liu, Z.; Wang, Z.; Li, G. Hybrid approach for detecting and classifying power quality disturbances based on the variational mode decomposition and deep stochastic configuration network. Energies 2018, 11, 3040. [CrossRef]

17. Rogers, G. Power System Oscillations; Kluwer: Boston, MA, USA, 2000.

18. Salford Systems, CART. 2008. Available online: www.salford-systems.com (accessed on 12 May 2008).

19. Fisher, R. The use of multiple measurements in taxonomic problems. Ann. Eugen. 1936, 7, 179-188. [CrossRef]

20. He, J.; Lu, C.; Wu, X.; Li, P.; Wu, J. Design and experiment of wide area HVDC supplementary damping controller considering time delay in China southern power grid. IET Gener. Transm. Distrib. 2009, 3, 17-25. [CrossRef]

21. Wang, J.; Fu, C.; Zhang, Y. Design of WAMS-based multiple HVDC damping control system. IEEE Trans. Smart Grid 2011, 2, 363-374. [CrossRef]

22. Huo, J.; Xue, A.; Wang, Q.; Bi, T.; Li, C. Research on clustering algorithm for dynamic equivalence of doubly-fed wind farms. In Proceedings of the 2014 International Conference on Power System Technology, Chengdu, China, 20-22 October 2014.

23. Yi, D.; Liu, C.; Tian, X. The power output characteristics of Jiuquan wind power base and its reactive power compensation. In Proceedings of the 2013 IEEE PES Asia-Pacific Power and Energy Engineering Conference, Kowloon, China, 8-11 December 2013.

24. Bernabeu, E. Methodology for a Security-Dependability Adaptive Protection Scheme Based on Data Mining. Ph.D. Thesis, Department of Electrical and Computer Engineering, Virginia Polytechnic Institute and State University, Blacksburg, VA, USA, 2009. 